\title{
A note on typographical errors defining Theodorsen's coefficients for aeroelastic analysis
}

2018, Vol. 24(10) 2061-2069

(C) The Author(s) 2016

Reprints and permissions: sagepub.co.uk/journalsPermissions.nav DOI: 10.1 177/1077546316677/9| journals.sagepub.com/home/jvc

SAGE

\author{
Douglas D Bueno' and Paulo J Paupitz Gonçalves ${ }^{2}$
}

\begin{abstract}
The problem of flutter is an important topic involving aeroelastic analysis. The first approach to studying this problem was proposed by Theodore Theodorsen in 1935 and, since that publication, Theodorsen's theory has been extensively used by many researchers. However, it is common to find typographical errors in the definitions of the coefficients introduced by Theodorsen to determine the aerodynamic forces in a typical section airfoil. In this context, this paper summarizes the most common typographical errors found in the literature and includes mathematical demonstrations to clarify the correct form of those coefficients.
\end{abstract}

\section{Keywords}

Aeroelasticity, flutter, Theodorsen's theory, typographical errors, Theodorsen's coefficients

\section{Introduction}

Theodorsen (1935) proposed a theory based on potential flow and the Kutta conditions for the study of aerodynamic instability and the mechanism of flutter. That work is, probably, the base for the majority of methods used in aeroelasticity analysis nowadays. Theodorsen's work was developed for an oscillating airfoil or airfoil-aileron with three degrees of freedom; his solution used certain definite integrals identified as Bessel functions of the first and second kind of zero and first order.

To compute unsteady aerodynamic forces on an airfoil section with three degrees of freedom, Theodorsen used various coefficients $T_{i}$, which are dependent on the geometry of the system. Although a detailed formulation had been presented (Theodorsen, 1935), the algebraic development of every coefficient was not presented. In this report, however, Theodorsen, developed illustrative examples and experimental results that validated his ideas.

Recently, authors have shown that Theodorsen's report was published with numerical errors. For example, Zeiler (2000) found a number of erroneous plots. However, Zeiler highlighted the historical importance of that work and also its usefulness in this field. After Zeiler's work, Perry (2015) recomputed the numerical results of Theodorsen's report (Theodorsen, 1935). Perry also published an engineering note to make this point clear (Perry, 2016). Zeiler and Perry found incorrect computations without any indication of what might be typographical errors.

Since Theodorsen's work was published, his theory has been used by researchers to develop methodologies in linear and nonlinear aeroelasticity and a large number of works have been published by the scientific community (Alighanbari, 1995; Ko et al., 1998; Bueno et al., 2014; Zhang and Behal, 2016). Among these works, several papers were disclosed with typographical errors in Theodorsen's coefficients (Edwards et al., 1979; Karpel, 1981; Kanda and Dowell, 2005; Li et al., 2010; Vasconcellos et al., 2012; Abdelkefi et al., 2012). The quality of these works is not questionable. However, particularly for students making initial steps

'Department of Mathematics, São Paulo State University, Ilha Solteira, Brazil

${ }^{2}$ Department of Mechanical Engineering, São Paulo State University, Bauru, Brazil

Received: 3 May 2016; accepted: 24 September 2016

Corresponding author:

Douglas D Bueno, Av. Brasil 56, São Paulo State University, Ilha Solteira, São Paulo I5385000 Brazil.

Email: douglas@mat.feis.unesp.br 


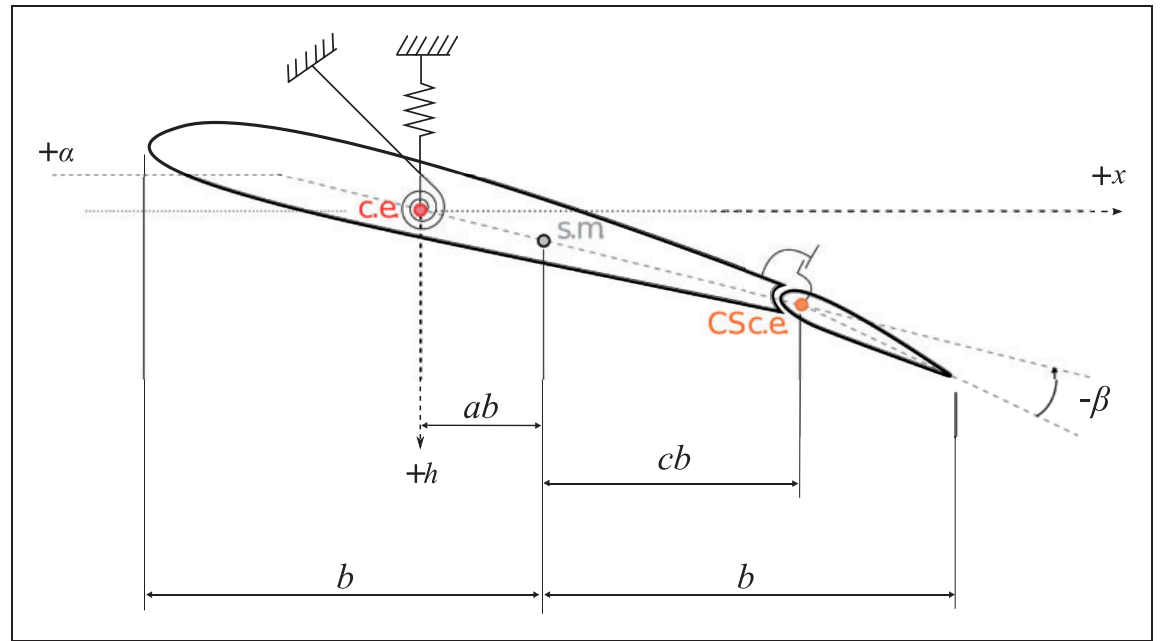

Figure I. Typical section airfoil.

in this area, these errors have have contributed to an increased in time wasted by comparing different bibliographical references. In addition, it is also reasonable to assume that some students might even be wasting some part of their time with incorrect computational implementations.

Typographical errors in scientific papers can eventually be found. For example, in the section "differential equation of motion" on page 419 of his report (Theodorsen, 1935), Theodorsen writes the term $\left(h S_{\beta}\right)$ instead of including the second derivative $\left(\ddot{h} S_{\beta}\right)$ for the rearranged equation of $(\beta)$. Similarly, equation $(C)$ on page 420 of the same report presents the term $[h(1+\kappa) /$ $b]$ instead of $[\ddot{h}(1+\kappa) / b]$.

Some typographical errors are not easily noticed while reading a paper. Some of them can probably be found during computational implementation, particularly for those errors where the results are significantly modified in comparison with expected results. Conversely, for the cases where the final results are unknown, the detection of a typographical error can be a hard task.

Several textbooks have introduced new ideas in aeroservoelasticity fields (Bisplinghoff and Ashley, 1962; Dowell et al., 1995; Hodges and Pierce, 2002; Wright and Cooper, 2007). Some of these also present complementary information and discuss applications involving Theodorsen's theory. However, algebraic demonstrations of each coefficient $T_{i}$ are commonly not included. In this context, the contribution of this paper is to present the most common typographical errors found in the literature, and clarify by analytical development the correct expressions that define them. In addition, this paper includes appendix I that defines the aerodynamic force and moments in matrix form, as they are often used by researchers.

\section{The most common typographical errors}

Theodorsen's theory, developed to study the mechanism of flutter, was based on a simple aeroelastic system, which represents a typical section airfoil. An illustrative scheme of this system is shown in Figure 1, where $h$ is the plunge, $\alpha$ is the pitch, $\beta$ is the control surface angular displacement, and $b$ is the aerodynamic semi-chord (s.m.). In this figure, c.e. denotes the elastic center, $a$ is the location of the elastic center measured from s.m., $c$ is the location of the control surface elastic center measured from s.m., and CS denotes the control surface. This illustration is important for understanding the physical meaning behind some of the equations presented in this paper.

By comparing relevant works from the literature, it is possible to verify that the coefficients $T_{4}, T_{13}, T_{9}, T_{7}$, and $T_{1}$ are often defined by different equations. It is presumed that these differences simply correspond to typographical errors. However, they can be found in doctoral theses, master's dissertations, and important papers published in specialized periodical journals and conferences.

According to Theodorsen (1935), each coefficient $T_{i}$ is obtained from velocity potentials and their analytical forms are considered herein as hypotheses to be confirmed. Based on a literature review, the most common typographical errors involving these coefficients are summarized in Table 1.

\section{I. Analytical development of some Theodorsen's coefficients}

In this section, algebraic demonstrations of each coefficient presented in Table 1 are developed with the 
Table I. The most common typographical errors for the definitions of Theodorsen's coefficients.

\begin{tabular}{ll}
\hline Coefficient & Most common error \\
\hline$T_{1}$ & It is written as a sum of two positive terms. \\
$T_{4}$ & It is not written as a sum of two terms. \\
$T_{7}$ & It is written as a sum of two negative terms. \\
$T_{9}$ & It is involved to define $T_{13}$. \\
$T_{11}$ & It is written with $\left[-\cos ^{-1}(c)(I-2 c)\right]$ \\
& instead of $\left[\cos ^{-1}(c)(I-2 c)\right]$. \\
$T_{13}$ & It appears multiplied by $-I$ instead of -2 \\
$M_{n c}$ & in matrix $\mathbf{M}_{n c}$. \\
$M_{n c}$ & Elements $(2,3)$ and $(3,2)$ are defined by $-T_{13}$ \\
& Element $(3,3)$ is defined by $T_{19} / \pi$ instead of \\
\end{tabular}

objective of comparing the analytical form with the results proposed by Theodorsen (1935). The idea herein is to clarify the correct definitions and the recursive forms used by Theodorsen, since the equations described by Theodorsen cannot be directly obtained from the solutions of the integrals. Probably, at that time, the recursive form was a convenient approach to minimize the calculations that were performed by hand. To demonstrate the correct form of some coefficients $T_{i}$, the integrals proposed by Theodorsen are considered as hypotheses to be confirmed.

\section{I.I. Coefficient $\mathrm{T}_{4}$.}

\section{Hypothesis from Theodorsen's work.}

$$
I=\int_{c}^{1} \varphi_{\theta} \mathrm{d} x=-\frac{1}{2} v \theta T_{4} \quad \text { and } \quad \varphi_{\theta}=v \theta b \sqrt{1-x^{2}}
$$

\section{Mathematical proof.}

$$
I=\int_{c}^{1} v \theta b \sqrt{1-x^{2}} \mathrm{~d} x
$$

Assume that $x=\cos (\lambda) \Rightarrow \mathrm{d} x=-\sin (\lambda) \mathrm{d} \lambda \quad$ and $\sqrt{1-x^{2}}=\sin (\lambda)$. Then, substituting the terms

$$
\begin{aligned}
I & =-v \theta b \int_{\cos ^{-1}(c)}^{0} \sin ^{2}(\lambda) \mathrm{d} \lambda \\
& =-\frac{1}{2} v \theta b \int_{\lambda_{1}}^{\lambda_{2}}[1+\cos (2 \lambda)] \mathrm{d} \lambda \\
& =-\frac{1}{2} v \theta b\left[\lambda-\frac{1}{2} \sin (2 \lambda)\right]_{\lambda_{1}}^{\lambda_{2}}
\end{aligned}
$$

$$
\begin{aligned}
& =-\frac{1}{2} v \theta b\left\{\cos ^{-1}(x)-\frac{1}{2} \sin \left[2 \cos ^{-1}(x)\right]\right\}_{c}^{1} \\
& =-\frac{1}{2} v \theta b\left[-\cos ^{-1}(c)+2 \frac{1}{2} \sqrt{1-c^{2}}\right]
\end{aligned}
$$

By comparing this result with equation (1), it is possible to prove that

$$
T_{4}=-\cos ^{-1}(c)+c \sqrt{1-c^{2}}
$$

\subsubsection{Coefficient $T_{9}$.}

\section{Hypothesis from Theodorsen's work.}

$$
I=\int_{c}^{1} \varphi_{\dot{\theta}} \mathrm{d} x=\dot{\theta} b^{2} T_{9}
$$

and

$$
\varphi_{\dot{\theta}}=\dot{\theta} b^{2}\left(\frac{1}{2} x-a\right) \sqrt{1-x^{2}}
$$

\section{Mathematical proof.}

$$
I=\dot{\theta} b^{2}\left[\frac{1}{2} \int_{c}^{1} x \sqrt{1-x^{2}} \mathrm{~d} x-a \int_{c}^{1} \sqrt{1-x^{2}} \mathrm{~d} x\right]
$$

From the constant $T_{4}$ note that

$$
\int_{c}^{1} \sqrt{1-x^{2}} \mathrm{~d} x=-\frac{1}{2} T_{4}
$$

Integrating separately, if $\left(1-x^{2}\right)=\lambda, \quad$ then $\mathrm{d} \lambda=-2 x \mathrm{~d} x$ and

$$
x \mathrm{~d} x=-\frac{1}{2} \mathrm{~d} \lambda
$$

Thus

$$
\begin{aligned}
\int_{c}^{1} x \sqrt{1-x^{2}} \mathrm{~d} x & =-\frac{1}{2} \int_{\lambda_{1}}^{\lambda_{2}} \sqrt{\lambda} \mathrm{d} \lambda=-\left.\frac{1}{2} \frac{2}{3}\left(\lambda^{3 / 2}\right)\right|_{\lambda_{1}} ^{\lambda_{2}} \\
& =\frac{1}{3}\left(\sqrt{1-c^{2}}\right)^{3}
\end{aligned}
$$

Then, denoting

$$
p=-\frac{1}{3}\left(1-c^{2}\right)^{3}
$$


and substituting the terms

$$
I=\dot{\theta} b^{2}\left[\frac{1}{2}(-p)+\frac{a}{2} T_{4}\right]
$$

By comparing this result with equation (2), it is possible to prove that

$$
T_{9}=\frac{1}{2}\left(-p+a T_{4}\right)
$$

\section{I.3. Coefficient $T_{1}$.}

Hypothesis from Theodorsen's work.

$$
I=\int_{c}^{1} \varphi_{\theta}(x-c) \mathrm{d} x=-\frac{b}{2} v \theta T_{1}
$$

\section{Mathematical proof.}

$$
I=v \theta b\left[\int_{c}^{1} x \sqrt{1-x^{2}} \mathrm{~d} x-c \int_{c}^{1} \sqrt{1-x^{2}} \mathrm{~d} x\right]
$$

From the constants $T_{4}$ and $T_{9}$, respectively, note that

$$
\int_{c}^{1} \sqrt{1-x^{2}} \mathrm{~d} x=-\frac{1}{2} T_{4}
$$

and

$$
\int_{c}^{1} x \sqrt{1-x^{2}} \mathrm{~d} x=\frac{1}{3}\left(\sqrt{1-c^{2}}\right)^{3}=\frac{1}{3} \sqrt{1-c^{2}}\left(1-c^{2}\right)
$$

Substituting the terms

$$
\begin{aligned}
& I=v \theta b\left\{\frac{1}{3} \sqrt{1-c^{2}}\left(1-c^{2}\right)-c \frac{1}{2}\left[\cos ^{-1}(c)-c \sqrt{1-c^{2}}\right]\right\} \\
&=v \theta b\left\{\frac{1}{3} \sqrt{1-c^{2}}-\frac{1}{3} c^{2} \sqrt{1-c^{2}}\right. \\
& \\
&\left.\quad+\frac{1}{2} c^{2} \sqrt{1-c^{2}}-\frac{c}{2} \cos ^{-1}(c)\right\} \\
&=v \theta b\left[\frac{1}{3} \sqrt{1-c^{2}}+\frac{1}{6} c^{2} \sqrt{1-c^{2}}-\frac{c}{2} \cos ^{-1}(c)\right] \\
&=v \theta b\left[\frac{1}{3} \sqrt{1-c^{2}}\left(1+\frac{1}{2} c^{2}\right)-\frac{c}{2} \cos ^{-1}(c)\right] \\
&=-\frac{1}{2} v \theta b\left[-\frac{1}{3} \sqrt{1-c^{2}}\left(2+c^{2}\right)+c \cos ^{-1}(c)\right]
\end{aligned}
$$

By comparing this result with equation (3), it is possible to prove that

$$
T_{1}=-\frac{1}{3} \sqrt{1-c^{2}}\left(2+c^{2}\right)+c \cos ^{-1}(c)
$$

\section{I.4. Coefficients $T_{7}$ and $T_{13}$.}

\section{Hypothesis from Theodorsen's work.}

$$
I=\int_{c}^{1} \varphi_{\dot{\theta}}(x-c) \mathrm{d} x=\dot{\theta} b^{2} T_{13}
$$

and

$$
T_{7}=-\left(\frac{1}{8}+c^{2}\right) \cos ^{-1}(c)+\frac{1}{8} c \sqrt{1-c^{2}}\left(7+2 c^{2}\right)
$$

Mathematical proof.

$$
\begin{array}{r}
I=\dot{\theta} b^{2}\left[\int_{c}^{1}\left(\frac{1}{2} x-a\right) x \sqrt{1-x^{2}} \mathrm{~d} x\right. \\
\left.\quad-c \int_{c}^{1}\left(\frac{1}{2} x-a\right) \sqrt{1-x^{2}} \mathrm{~d} x\right]
\end{array}
$$

Writing $I=\dot{\theta} b^{2}\left[I_{1}+I_{2}+I_{3}+I_{4}\right]$ to simplify the notation, it is possible to evaluate each term separately

$$
\begin{aligned}
I_{1} & =\frac{1}{2} \int_{c}^{1} x^{2} \sqrt{1-x^{2}} \mathrm{~d} x \Rightarrow \text { if } x=\cos (\lambda) \\
& =-\frac{1}{2} \int_{\lambda_{1}}^{\lambda_{2}} \cos ^{2}(\lambda) \sin ^{2}(\lambda) \mathrm{d} \lambda \\
& =-\frac{1}{2} \int_{\lambda_{1}}^{\lambda_{2}}\left[\frac{1+\cos (2 \lambda)}{2}\right]\left[\frac{1-\cos (2 \lambda)}{2}\right] \mathrm{d} \lambda \\
& =-\frac{1}{8} \int_{\lambda_{1}}^{\lambda_{2}}\left[1-\cos ^{2}(2 \lambda)\right] \mathrm{d} \lambda \\
& =-\frac{1}{8}\left\{\int_{\lambda_{1}}^{\lambda_{2}} \mathrm{~d} \lambda-\int_{\lambda_{1}}^{\lambda_{2}}\left[\frac{1+\cos (4 \lambda)}{2}\right] \mathrm{d} \lambda\right\} \\
& =-\frac{1}{8}\left\{\left.\lambda\right|_{\lambda_{1}} ^{\lambda_{2}}-\frac{1}{2}\left[-\cos ^{-1}(c)+\left.\frac{1}{4} \sin (4 \lambda)\right|_{\lambda_{1}} ^{\lambda_{2}}\right]\right\}
\end{aligned}
$$

where $\lambda_{1}=\cos ^{-1}(c), \lambda_{2}=0$, and

$$
\begin{aligned}
\sin (4 \lambda) & =2 \sin (2 \lambda) \cos (2 \lambda) \\
& =2[2 \sin (\lambda) \cos (\lambda)]\left[\cos ^{2}(\lambda)-\sin ^{2}(\lambda)\right]
\end{aligned}
$$

If $\quad x=c, \quad \sin (\lambda)=\sqrt{1-\cos ^{2}(\lambda)}, \quad$ and $\quad \sin ^{2}(\lambda)=$ $\cos ^{2}(\lambda)-1$, then $\sin (4 \lambda)=4 c \sqrt{1-c^{2}}\left(2 c^{2}-1\right)$. Substituting the terms in $I_{1}$

$$
I_{1}=-\frac{1}{8}\left[-\frac{1}{2} \cos ^{-1}(c)+\frac{c}{2} \sqrt{1-c^{2}}\left(2 c^{2}-1\right)\right]
$$


Similarly, it is possible to write the terms based on previous demonstrations, i.e.

$$
\begin{aligned}
& I_{2}=-a \int_{c}^{1} x \sqrt{1-x^{2}} \mathrm{~d} x=-\frac{a}{3}\left(\sqrt{1-c^{2}}\right)^{3} \\
& I_{3}=-c \frac{1}{2} \int_{c}^{1} x \sqrt{1-x^{2}} \mathrm{~d} x=-\frac{c}{6}\left(\sqrt{1-c^{2}}\right)^{3} \\
& I_{4}=-\frac{a c}{2}\left[-\cos ^{-1}(c)+c \sqrt{1-c^{2}}\right]
\end{aligned}
$$

At this point, the integral $I$ defined in equation (4) is solved and it can be computed by $\dot{\theta} b^{2} \sum_{i=1}^{4} I_{i}$. However, it is not yet possible to compare the results explicitly. Thus, a nonintuitive rearrangement is presented as

$$
\begin{aligned}
\sum_{i=1}^{4} I_{i}+\sum_{j=1}^{4} \bar{I}_{j}= & {\left[+\frac{1}{8} \frac{\cos ^{-1}(c)}{2}-\frac{1}{8} \frac{c}{2} \sqrt{1-c^{2}}\left(2 c^{2}-1\right)\right] } \\
& +\left[-\left(\frac{c}{2}+a\right) \frac{1}{3} \sqrt{1-c^{2}}\left(1-c^{2}\right)\right] \\
& +\left[\frac{a c}{2} \cos ^{-1}(c)-\frac{1}{2} a c^{2} \sqrt{1-c^{2}}\right]+\sum_{j=1}^{4} \bar{I}_{j}
\end{aligned}
$$

where

$$
\begin{aligned}
\sum_{j=1}^{4} \bar{I}_{j}= & \left(\frac{c^{2} \cos ^{-1}(c)}{2}-\frac{c^{2} \cos ^{-1}(c)}{2}\right) \\
& +\left(\frac{c \sqrt{1-c^{2}}}{2}-\frac{c \sqrt{1-c^{2}}}{2}\right)=0
\end{aligned}
$$

and, then

$$
\begin{aligned}
\sum_{i=1}^{4} I_{i} & +\sum_{j=1}^{4} \bar{I}_{j} \\
= & {\left[\frac{1}{2}\left(\frac{1}{8}+c^{2}\right) \cos ^{-1}(c)\right] } \\
+ & {\left[-\frac{1}{2} \frac{1}{8} c \sqrt{1-c^{2}}\left(7+c^{2}\right)\right] } \\
+ & {\left[-a \frac{2}{2} \frac{1}{3} \sqrt{1-c^{2}}+a c^{2} \frac{1}{3} \sqrt{1-c^{2}}\right.} \\
& \left.-\frac{c}{2} \frac{1}{3} \sqrt{1-c^{2}}\left(1-c^{2}\right)\right] \\
+ & {\left[+\frac{1}{2} a c \cos ^{-1}(c)-\frac{1}{2} a c^{2} \sqrt{1-c^{2}}\right] } \\
+ & \left(-\frac{c^{2} \cos ^{-1}(c)}{2}+\frac{c \sqrt{1-c^{2}}}{2}\right)
\end{aligned}
$$

$$
\begin{aligned}
& \sum_{i=1}^{4} I_{i}+\sum_{j=1}^{4} \bar{I}_{j} \\
&=- \frac{1}{2}\left[-\left(\frac{1}{8}+c^{2}\right) \cos ^{-1}(c)+\frac{1}{8} c \sqrt{1-c^{2}}\left(7+c^{2}\right)\right] \\
&+ \frac{1}{2} a\left[-\frac{2}{3} \sqrt{1-c^{2}}-c^{2} \sqrt{1-c^{2}}\right. \\
&\left.+c^{2} \frac{2}{3} \sqrt{1-c^{2}}+c \cos ^{-1}(c)\right] \\
&- \frac{c}{2}\left[\frac{1}{3} \sqrt{1-c^{2}}\left(1-c^{2}\right)+c \cos ^{-1}(c)-\sqrt{1-c^{2}}\right] \\
& \sum_{i=1}^{4} I_{i}+\sum_{j=1}^{4} \bar{I}_{j} \\
&=-\frac{1}{2}\left[-\left(\frac{1}{8}+c^{2}\right) \cos ^{-1}(c)+\frac{1}{8} c \sqrt{1-c^{2}}\left(7+c^{2}\right)\right] \\
&+\frac{1}{2} a\left[-\frac{1}{3} \sqrt{1-c^{2}}\left(2+c^{2}\right)+c \cos ^{-1}(c)\right] \\
&-\frac{c}{2}\left[-\frac{1}{3} \sqrt{1-c^{2}}\left(2+c^{2}\right)+c \cos ^{-1}(c)\right]
\end{aligned}
$$

Considering the definition of $T_{1}$, it is possible to write

$$
\begin{aligned}
\sum_{i=1}^{4} I_{i} & +\sum_{j=1}^{4} \bar{I}_{j} \\
= & -\frac{1}{2}\left[-\left(\frac{1}{8}+c^{2}\right) \cos ^{-1}(c)+\frac{1}{8} c \sqrt{1-c^{2}}\left(7+c^{2}\right)\right] \\
& +\frac{1}{2} a T_{1}-\frac{c}{2} T_{1}=\sum_{i=1}^{4} I_{i} \\
\sum_{i=1}^{4} I_{i}= & \frac{1}{2}\left\{-\left[-\left(\frac{1}{8}+c^{2}\right) \cos ^{-1}(c)+\frac{1}{8} c \sqrt{1-c^{2}}\left(7+c^{2}\right)\right]\right. \\
& \left.-(c-a) T_{1}\right\}
\end{aligned}
$$

By comparing this result with equation (4), it is possible to prove that if

$$
T_{7}=-\left(\frac{1}{8}+c^{2}\right) \cos ^{-1}(c)+\frac{1}{8} c \sqrt{1-c^{2}}\left(7+c^{2}\right)
$$

then

$$
T_{13}=\frac{1}{2}\left[-T_{7}-(c-a) T_{1}\right]
$$




\section{Discussion of results}

To demonstrate the influence of the typographic errors and their effect on the final solutions of a certain aeroelastic problem is not a trivial task, since the final results depend on various other parameters (structural properties, and the combination of several errors simultaneously). However, it is possible to present a simplified discussion to clarify how a simple mistake can affect the unsteady aerodynamic loads proposed by Theodorsen.

Considering the system presented in Figure 1 for the case in which both pitch and plunge degrees of freedom are fixed, i.e.

$$
h(t)=\theta(t)=0
$$

the Theodorsen's force presented in equation (8) can be simplified to $\tilde{F}_{h}$, such that

$$
\begin{aligned}
\tilde{F}_{h}= & -\rho b^{2}\left(-V T_{4} \dot{\beta}-T_{1} b \ddot{\beta}\right) \\
& -2 \pi \rho V b C\left[\frac{1}{\pi} T_{10} V \beta+b \frac{1}{2 \pi} T_{11} \dot{\beta}\right]
\end{aligned}
$$

Assuming the control surface displacement $\beta=\beta_{0}$ $\sin (\phi)$ (where $\phi=\omega t$ ), and rewriting $\phi$ in terms of the reduced frequency

$$
\begin{aligned}
& \beta_{\mathrm{cy}}(\phi)=\beta_{0} \sin (\phi) \\
& \dot{\beta}_{\mathrm{cy}}(\phi)=\beta_{0}\left(\frac{V}{b} k\right) \cos (\phi) \\
& \ddot{\beta}_{\mathrm{cy}}(\phi)=-\beta_{0}\left(\frac{V}{b} k\right)^{2} \sin (\phi)
\end{aligned}
$$

where the angle $\phi$ is an angular displacement and subscript "cy" indicates one cycle of motion $(0 \leq \phi \leq 2 \pi)$. Using equation (7), the unsteady aerodynamic force (equation (6)) can be determined.

The following results of $\tilde{F}_{h}$ were obtained considering the correct form of $T_{1}$ (shown in Appendix 2) in comparison with its incorrect form, computed as indicated in the first line of Table 1. The parameters used in the analysis were

$$
\begin{aligned}
b & =0.15 \mathrm{~m} \\
\rho & =1.225 \mathrm{~kg} / \mathrm{m}^{3} \\
V & =1 \mathrm{~m} / \mathrm{s} \\
c & =0.25 \\
\beta_{0} & =3 \pi / 180 \mathrm{rad}
\end{aligned}
$$

Figure 2 shows how the real and imaginary parts of $\tilde{F}_{h}$ differ from the correct forms. Clearly, it can be noted that a simple mistake results in very different values for

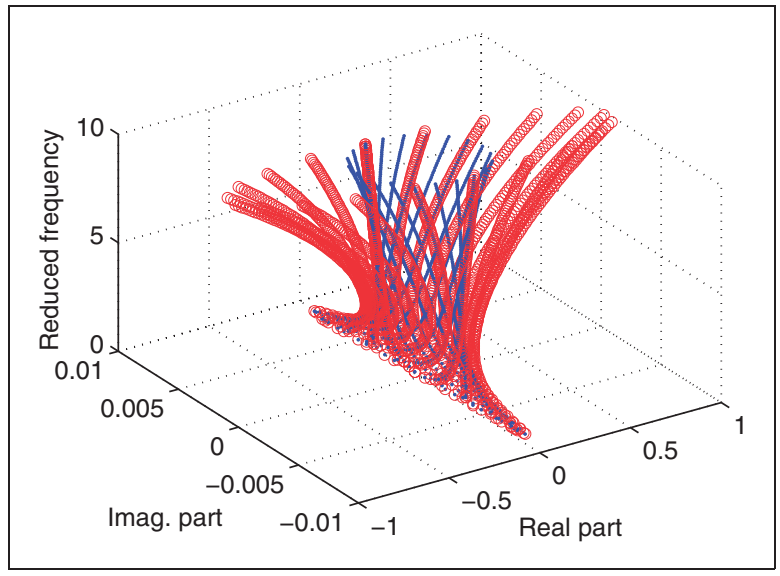

Figure 2. Real and imaginary parts of $\tilde{F}_{h}$. Blue dots represent correct values and red circles represent incorrect values.

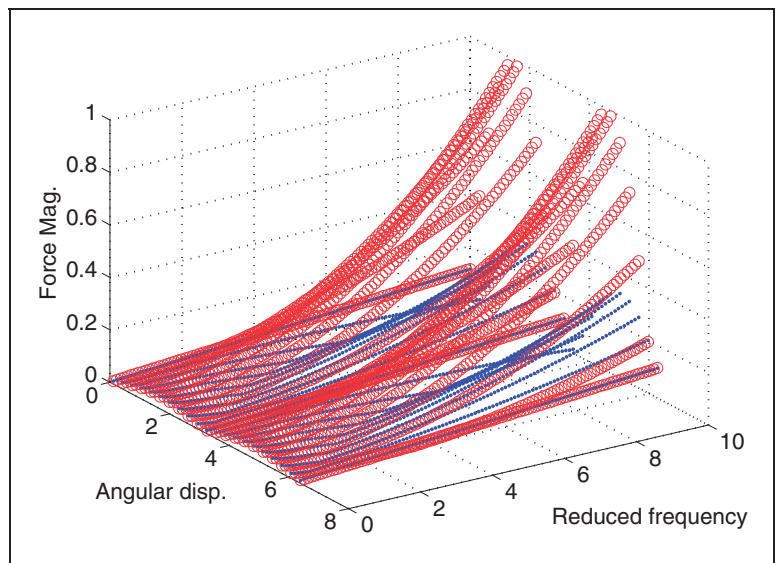

Figure 3. Magnitude of $\tilde{F}_{h}$ over a cycle for different values of $k$. Blue dots represent correct values and red circles represent incorrect values.

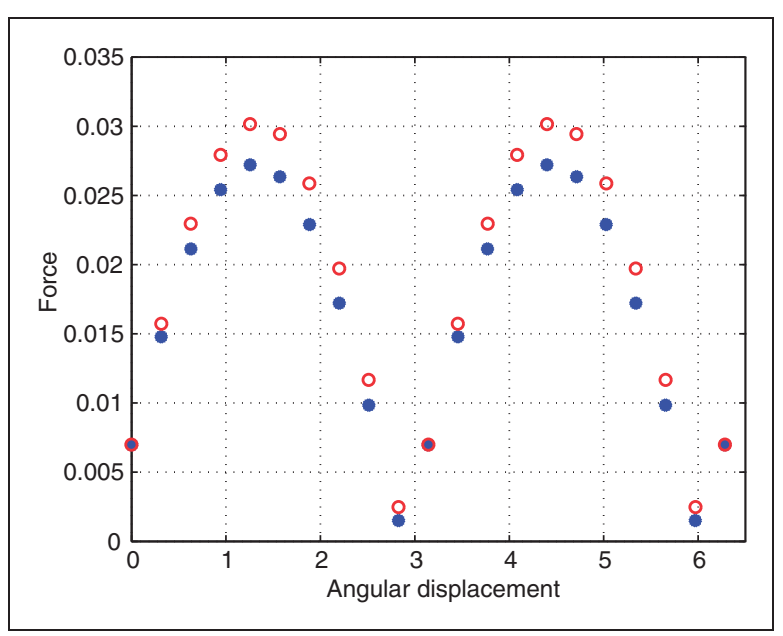

Figure 4. Magnitude of $\tilde{F}_{h}$ for $k=0.5$. Blue dots represent correct values and red circles represent incorrect values. 


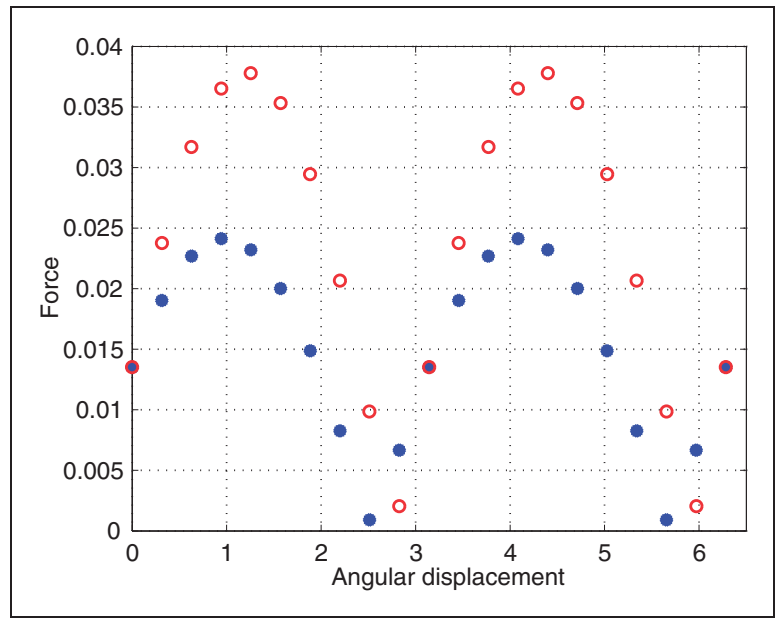

Figure 5. Magnitude of $\tilde{F}_{h}$ for $k=I$.I. Blue dots represent correct values and red circles represent incorrect values.

high values of reduced frequency. These results can also be verified from equation (6) (or equation (8)), which shows the coefficient $T_{1}$ multiplied by $\ddot{\beta}$, since the latter is a function of $k^{2}$.

The presented differences also imply an incorrect magnitude of the unsteady aerodynamic force. According to Figure 3, the value of $\left|\tilde{F}_{h}\right|$ over a cycle of motion is more inaccurate for high reduced frequencies. However, small values, e.g. $k=0.5$ (Figure 4) and $k=1.1$ (Figure 5) can also result on incorrect computations. The results shown in Figure 5 also indicate an important error in the phase of $\tilde{F}_{h}$.

\section{Final remarks}

Since its publication in 1935, Theodorsen's theory has established the foundations of aeroelasticity. The aerodynamic forces for a typical section airfoil were defined in terms of coefficients for which Theodorsen's report (Theodorsen (1935)) does not present a complete mathematical demonstration. Some of these coefficients cannot be defined directly by solving an integral because they require recursive solutions to be explicitly determined. In this context, this paper presented the most common typographical errors found in the definition of these coefficients. The corrected coefficients were calculated and are listed in Appendix 2 for easy reference.

\section{Declaration of Conflicting Interests}

The author(s) declared no potential conflicts of interest with respect to the research, authorship, and/or publication of this article.

\section{Funding}

The author(s) received no financial support for the research, authorship, and/or publication of this article.

\section{References}

Abdelkefi A, Vasconcellos R, Marques FD, et al. (2012) Bifurcation analysis of an aeroelastic system with concentrated nonlinearities. Nonlinear Dynamics 69: 57-70.

Alighanbari H (1995) Flutter analysis and chaotic response of an airfoil accounting for structural nonlinearities. $\mathrm{PhD}$ Thesis, McGill University, Canada.

Bisplinghoff R and Ashley H (1962) Principles of Aeroelasticity. New York: Wiley.

Bueno DD, Góes LCS and Gonçalves PJP (2014) Control of limit cycle oscillation in a three degrees of freedom airfoil section using fuzzy Takagi-Sugeno modeling. Shock and Vibration 2014: 1-12.

Dowell EH, Crawley EF, Curtiss Jr HC, et al. (1995) $A$ Modern Course in Aeroelasticity. Dordrecht: Kluwer Academic Publishers.

Edwards J, Ashley H and Breakwell J (1979) Unsteady aerodynamic modeling for arbitrary motions. AIAA Journal 17(4): 365-374.

Hodges DH and Pierce GA (2002) Introduction to Structural Dynamic and Aeroelasticity. New York: Cambridge University Press.

Kanda A and Dowell EH (2005) Worst-case gust-response analysis for typical airfoil section with control surface. Journal of Aircraft 42(4): 956-962.

Karpel M (1981) Design for active and passive flutter suppression and gust alleviation. Report no. TR 3482, National Aeronautics and Space Administration, Washington DC.

Ko J, Strganac TW, and Kurdila AJ (1998) Nonlinear adaptive control of an aeroelastic system via geometric methods. In: 39th AIAA/ASME/ASCE/AHS/ASC structures, structural dynamics, and material conference, Long Beach, CA, 20-23 April 1998, pp.98-1795. Reston, VA: AIAA.

Li D, Guo S and Xiang J (2010) Aeroelastic dynamic response and control of an airfoil section with control surface nonlinearities. Journal of Sound and Vibration 329(22): 4756-4771.

Perry B (2015) Re-computation of numerical results contained in NACA report no. 496. Technical report, NASA Langley Research Center, Hampton, VA.

Perry B (2016) Results of national advisory committee for aeronautics report number 496: revisited. Journal of Aircraft 53(5): 1561-1565.

Theodorsen T (1935) General theory of aerodynamic instability and the mechanism of flutter. NACA report no 496.

Vasconcellos R, Abdelkefi A, Marques FD, et al. (2012) Representation and analysis of control surface freeplay nonlinearity. Journal of Fluids and Structures 31: 79-91.

Wright JR and Cooper JE (2007) Introduction to Aircraft Aeroelasticity and Loads. Chichester: John Wiley \& Sons, Ltd.

Zeiler TA (2000) Results of Theodorsen and Garrick revisited. Journal of Aircraft 37(5): 918-920.

Zhang K and Behal A (2016) Continuous robust control for aeroelastic vibration control of a 2-D airfoil under unsteady flow. Journal of Vibration and Control 22(12): 2841-28620. 


\section{Appendix I. Aerodynamic force and moments}

According to Theodorsen, the total aerodynamic force and moments for each degree of freedom are given by

$$
\begin{aligned}
F_{h}= & -\rho b^{2}\left(V \pi \dot{\alpha}+\pi \ddot{h}-\pi b a \ddot{\alpha}-V T_{4} \dot{\beta}-T_{1} b \ddot{\beta}\right) \\
& -2 \pi \rho V b C\left[V \alpha+\dot{h}+b\left(\frac{1}{2}-a\right) \dot{\alpha}\right. \\
& \left.+\frac{1}{\pi} T_{10} V \beta+b \frac{1}{2 \pi} T_{11} \dot{\beta}\right] \\
M_{\alpha}= & -\rho b^{2}\left[\pi\left(\frac{1}{2}-a\right) V b \dot{\alpha}+\pi b^{2}\left(\frac{1}{8}-a^{2}\right) \ddot{\alpha}\right. \\
+ & \left(T_{4}+T_{10}\right) V^{2} \beta+\left(T_{1}-T_{8}-(c-a) T_{4}+\frac{1}{2} T_{11}\right) \\
& \left.\times V b \dot{\beta}-\left(T_{7}+(c-a) T_{1}\right) b^{2} \ddot{\beta}-a \pi b \ddot{h}\right] \\
+ & 2 \rho V b^{2} \pi\left(a+\frac{1}{2}\right) C\left[V \alpha+\dot{h}+b\left(\frac{1}{2}-a\right) \dot{\alpha}\right. \\
+ & \left.\frac{1}{\pi} T_{10} V \beta+b \frac{1}{2 \pi} T_{11} \dot{\beta}\right] \\
M_{\beta}= & -\rho b^{2}\left[\left(-2 T_{9}-T_{1}+T_{4}\left(a-\frac{1}{2}\right)\right) V b \dot{\alpha}+2 T_{13} b^{2} \ddot{\alpha}\right. \\
+ & \frac{1}{\pi} V^{2} \beta\left(T_{5}-T_{4} T_{10}\right)-\frac{1}{2 \pi} V b \dot{\beta} T_{4} T_{11} \\
& \left.-\frac{1}{\pi} T_{3} b^{2} \ddot{\beta}-T_{1} b \ddot{h}\right] \\
- & \rho V b^{2} T_{12} C\left[V \alpha+\dot{h}+b\left(\frac{1}{2}-a\right) \dot{\alpha}\right. \\
+ & \left.\frac{1}{\pi} T_{10} V \beta+b \frac{1}{2 \pi} T_{11} \dot{\beta}\right]
\end{aligned}
$$

where $\rho$ is the air density, $V$ is the airspeed, and $C$ is Theodorsen's function. Theodorsen (1935) presents these quantities considering the sequence $\alpha, \beta$, and $h$. However, in the literature, they are commonly organized in matrix form, considering the displacement vector explicitly defined by $\mathbf{u}=\{h \alpha \beta\}^{T}$.

\section{Al.I Aerodynamic forces in matrix form}

The general aerodynamic force in matrix form $\mathbf{F}_{a}(s)=$ $q \mathbf{Q}\left(s^{\prime}\right) \mathbf{u}(s)$ involves the matrix $\mathbf{Q}\left(s^{\prime}\right)$, also termed the generalized aerodynamic influence coefficient matrix, which is given by

$$
\begin{aligned}
\mathbf{Q}\left(s^{\prime}\right)= & 2 b^{2}\left[\mathbf{M}_{\mathrm{nc}}\left(s^{\prime}\right)^{2}+\left(\mathbf{B}_{\mathrm{nc}}+C\left(s^{\prime}\right) \mathbf{R}_{1} \mathbf{S}_{2}\right) s^{\prime}\right. \\
& \left.+\mathbf{K}_{\mathrm{nc}}+C\left(s^{\prime}\right) \mathbf{R}_{1} \mathbf{S}_{1}\right]
\end{aligned}
$$

where $s^{\prime}=\mathrm{i} k$ is the Laplace variable defined in terms of the reduced frequency $k, \mathrm{i}$ is the imaginary number, and the subscript "nc" indicates the noncirculatory part. The reduced frequency is computed by $k=\omega b / V$, where $\omega$ is the angular frequency in $\mathrm{rad} / \mathrm{s}$. The following matrices are obtained through a rearrangement of equations (8), (9), and (10), and considering the aeroelastic displacement vector equal to $\mathbf{u}=\{h \alpha \beta\}^{T}$

$$
\begin{gathered}
\mathbf{M}_{\mathrm{nc}}=\left[\begin{array}{ccc}
-\pi & \pi a & T_{1} \\
\pi a & -\pi\left(a^{2}+1 / 8\right) & -2 T_{13} \\
T_{1} & -2 T_{13} & T_{3} / \pi
\end{array}\right] \\
\mathbf{B}_{\mathrm{nc}}=\left[\begin{array}{ccc}
0 & -\pi a & T_{4} \\
0 & \pi(a-1 / 2) & -T_{16} \\
0 & -T_{17} & -T_{19} / \pi
\end{array}\right] \\
\mathbf{K}_{\mathrm{nc}}=\left[\begin{array}{ccc}
0 & 0 & 0 \\
0 & 0 & -T_{15} \\
0 & 0 & -T_{18} / \pi
\end{array}\right] \\
\mathbf{R}_{1}=\left[\begin{array}{lll}
-2 \pi & 2 \pi\left(a+\frac{1}{2}\right) & -T_{12}
\end{array}\right]^{\mathrm{T}} \\
\mathbf{S}_{1}=\left[\begin{array}{lll}
0 & 1 & T_{10} / \pi
\end{array}\right] \\
\mathbf{S}_{2}=\left[\begin{array}{lll}
1 & \left(\frac{1}{2}-a\right) & \frac{T_{11}}{2 \pi}
\end{array}\right]
\end{gathered}
$$

\section{Appendix 2. Theodorsen's coefficients}

Each coefficient can be computed using the following equations, where $a$ is the location of the elastic center, measured from the aerodynamic semi-chord $(b)$, and $c$ is the location of the control surface elastic center, measured from the aerodynamic semi-chord

$$
\begin{aligned}
T_{1}= & -\frac{1}{3} \sqrt{1-c^{2}}\left(2+c^{2}\right)+c \cos ^{-1}(c) \\
T_{2}= & c\left(1-c^{2}\right)-\sqrt{1-c^{2}}\left(1+c^{2}\right) \cos ^{-1}(c) \\
& +c\left(\cos ^{-1}(c)\right)^{2} \\
T_{3}= & -\left(\frac{1}{8}+c^{2}\right)\left(\cos ^{-1}(c)\right)^{2} \\
& +\frac{1}{4} c \sqrt{1-c^{2}} \cos ^{-1}(c)\left(7+2 c^{2}\right)-\frac{1}{8}\left(1-c^{2}\right)\left(5 c^{2}+4\right) \\
T_{4}= & -\cos ^{-1}(c)+c \sqrt{1-c^{2}} \\
T_{5}= & -\left(1-c^{2}\right)-\left(\cos ^{-1}(c)\right)^{2}+2 c \sqrt{1-c^{2}} \cos ^{-1}(c) \\
T_{6}= & T_{2} \\
T_{7}= & -\left(\frac{1}{8}+c^{2}\right) \cos ^{-1}(c)+\frac{1}{8} c \sqrt{1-c^{2}}\left(7+2 c^{2}\right)
\end{aligned}
$$




$$
\begin{aligned}
& T_{8}=-\frac{1}{3} \sqrt{1-c^{2}}\left(2 c^{2}+1\right)+c \cos ^{-1}(c) \\
& T_{9}=\frac{1}{2}\left[\frac{1}{3}\left(\sqrt{1-c^{2}}\right)^{3}+a T_{4}\right]=\frac{1}{2}\left(-p+a T_{4}\right)
\end{aligned}
$$

where

$$
\begin{aligned}
p & =-\frac{1}{3}\left(\sqrt{1-c^{2}}\right)^{3} \\
T_{10} & =\sqrt{1-c^{2}}+\cos ^{-1}(c) \\
T_{11} & =\cos ^{-1}(c)(1-2 c)+\sqrt{1-c^{2}}(2-c) \\
T_{12} & =\sqrt{1-c^{2}}(2+c)-\cos ^{-1}(c)(2 c+1) \\
T_{13} & =\frac{1}{2}\left[-T_{7}-(c-a) T_{1}\right] \\
T_{14} & =\frac{1}{16}+\frac{1}{2} a c \\
T_{15} & =T_{4}+T_{10}
\end{aligned}
$$

$$
\begin{aligned}
& T_{16}=T_{1}-T_{8}-(c-a) T_{4}+\frac{T_{11}}{2} \\
& T_{17}=-2 T_{9}-T_{1}+\left(a-\frac{1}{2}\right) T_{4} \\
& T_{18}=T_{5}-T_{4} T_{10} \\
& T_{19}=-\frac{1}{2} T_{4} T_{11}
\end{aligned}
$$

